\title{
Hacia un abordaje multidimensional y multiescalar de la cuestión ecológica: La perspectiva del buen vivir
}

Towards a Multidimensional and Multi-scale Approach of the Ecological Issue: The Perspective of Good Living

Vers un abordage multidimensionnel et multi-échelle de la question écologique: La perspective du "bon vivre"

Victoria Haidar y María Valeria Berros

\section{OpenEdition}

\section{Journals}

Edición electrónica

URL: http://journals.openedition.org/rccs/6133

DOI: $10.4000 /$ rccs. 6133

ISSN: 2182-7435

Editor

Centro de Estudos Sociais da Universidade de Coimbra

Edición impresa

Fecha de publicación: 1 diciembre 2015

Paginación: 111-134

ISSN: 0254-1106

Referencia electrónica

Victoria Haidar y María Valeria Berros, « Hacia un abordaje multidimensional y multiescalar de la cuestión ecológica: La perspectiva del buen vivir », Revista Crítica de Ciências Sociais [En línea] 108 | 2015, Puesto en línea el 16 diciembre 2015, consultado el 19 abril 2019. URL : http:// journals.openedition.org/rccs/6133; DOI : 10.4000/rccs.6133 


\section{Hacia un abordaje multidimensional y multiescalar de la cuestión ecológica: La perspectiva del buen vivir}

Este artículo propone reponer el carácter multidimensional y multiescalar que asume la cuestión ecológica en el interior de la propuesta del "buen vivir" en Ecuador y en Bolivia a partir de tres ejes: i) los múltiples significados sobre la naturaleza que conviven en las regulaciones y en los planes nacionales de ambos países, ii) la afirmación sobre la interdependencia y la complementariedad de los derechos reconocidos a la naturaleza con relación a los derechos individuales y colectivos, iii) la circulación de estas propuestas desde el territorio andino en América Latina hacia el espacio global.

Palabras clave: Bolivia; buen vivir; ecologia; Ecuador; protección del ambiente.

Palavras-chave: Bolívia; bom viver; ecologia; Equador; proteção do ambiente.

\section{Introducción}

Desde su instalación en la agenda de las organizaciones internacionales y de la progresiva conformación de un régimen global de gobernanza del ambiente, la cuestión ecológica ${ }^{1}$ se ha presentado como uno de los tópicos privilegiados de las encrucijadas que atraviesan a los países de América Latina, así como de las confrontaciones que estos mantienen con los países del "Norte". Así, ya en el marco de la convocatoria a la Conferencia sobre Medio Humano (1972), la tensión entre el "desarrollo" y la "protección del ambiente" aparecía vertebrando las polémicas entre quienes sostenían que la conservación del planeta demandaba el cese del crecimiento económico, y de aquellos que, por el contrario, pensaban que con la mediación de la tecnología ambos objetivos podían compatibilizarse. Esta controversia

\footnotetext{
${ }^{1}$ Entendida como el "haz de problemas" concernientes a las relaciones entre el desarrollo, la protección de la naturaleza y la pobreza.
} 
concernía particularmente a los países del Tercer $\mathrm{Mundo}^{2}$ porque las preocupaciones ecológicas implicaban un cuestionamiento de la legitimidad y la viabilidad (a largo plazo) del "desarrollo", que dichos países concebían como medio para resolver el problema de la pobreza.

A pesar de la impronta conciliadora y universalista que tradujo el modelo del "desarrollo sostenible", el objetivo de articular los vectores del desarrollo económico, la lucha contra la pobreza y la protección del ambiente, se ha visto confrontado, a lo largo de los años transcurridos desde el Informe "Nuestro Futuro Común" (1987) y el Documento Final de la Conferencia de Naciones Unidas sobre Desarrollo Sostenible, "El futuro que queremos" (2012), a toda una serie de cuestionamientos que traslucen la heterogeneidad del concepto de sustentabilidad, en el que coexisten, de manera contradictoria, perspectivas que enfatizan la satisfacción de las necesidades humanas de las actuales generaciones junto a otras preocupadas por las generaciones futuras; voces que priorizan, superponiéndose a otros propósitos, la preservación de la biodiversidad y el respeto por la capacidad de carga de los diversos ecosistemas; así como sectores que atienden prioritariamente al crecimiento económico capitalista y su viabilidad a largo plazo. En el marco de esa pluralidad conflictiva de visiones, los países del Sur no han dejado de interponer resistencias frente a los proyectos de establecer un régimen de gobernanza del ambiente que imponga, de manera uniforme, obligaciones ambientales a los Estados, sin atender a las profundas diferencias que los separan de aquellos del "Primer Mundo" y obliterando, asimismo, la existencia de relaciones de colonialismo. ${ }^{3}$

Así, la hegemonía que -entre las múltiples lecturas que habilita la noción de "sostenibilidad" - ha adquirido, en la última convención de la ONU, el enfoque de la economía verde ${ }^{4}$ ha motivado la articulación de una serie de críticas desde algunos países de América Latina que encuentran, en esas estrategias, un "nuevo colonialismo de doble partida" (Morales, 2012) que mercantiliza la naturaleza y moviliza argumentos ambientalistas con

\footnotetext{
${ }^{2}$ Si bien en la actualidad el par países "desarrollados" y países "en desarrollo" suele preferirse a la más antigua distinción entre países del "Primer Mundo" y países del "Tercer Mundo", a lo largo de este texto usamos la expresión "Tercer Mundo" para referirnos a un punto de vista político-cultural que comprende tanto Estados como movimientos sociales, al interior del cual se han articulado -de manera cambiante y contingente- una serie de problemas de un modo contrapuesto, diferente y/o alternativo, a aquel esgrimido desde el "Primer Mundo". En todo caso, dicha fórmula no alude, necesariamente, a los límites político-territoriales de los Estados, sino a aquellos que separan las formas de vida (Rajagopal, 2003: 42).

${ }^{3}$ Esas resistencias han conducido al establecimiento del principio de las responsabilidades comunes pero diferenciadas, en materia, por ejemplo, de la agenda del cambio climático.

${ }^{4}$ La literatura sobre green economy no es nueva, puede consultarse, entre otros trabajos: Jacobs (1991), Milani (2000).
} 
la finalidad de legitimar el dominio, histórico, que los países del Norte han ejercido sobre los del Sur. Las políticas inspiradas en la economía verde se articulan con el objetivo de construcción del desarrollo sostenible, concepto que viene siendo criticado desde múltiples ámbitos de manera reiterada. Asimismo, se lo presenta como una herramienta posible para la erradicación de la pobreza lo que ha quedado traducido, de manera reiterada, al interior del documento final de la Cumbre de Naciones Unidas. En este marco, conceptos como servicios ambientales, uso óptimo de los recursos naturales, management, generación de beneficios, se advierten medulares y se enhebran con un criterio de utilidad de base antropocéntrica que está siendo problematizado por parte de posiciones que tienden a colocar el acento en un tránsito hacia la desmercantilización de lo vivo. ${ }^{5}$

Planteamientos como los realizados por el presidente Evo Morales, que citamos más arriba, no resultan aislados, sino que se inscriben en el marco de un conjunto de procesos sociales y debates relativos al concepto -plural y en construcción (Acosta, 2012; Santos, 2010) - de "bien vivir/ buen vivir/ sumak kawsay", ${ }^{6}$ que vienen desarrollándose en Bolivia y Ecuador a partir de la recuperación, reformulación y desarrollo creativo de las cosmovisiones, reflexiones y prácticas procedentes de los pueblos indígenas andinos y en los que resuenan, asimismo, toda otra serie de "memorias discursivas" (Courtine, 1981). ${ }^{7}$

El "buen vivir" 8 ha sido puesto en discusión en el contexto de las luchas encabezadas por esos pueblos y diversos movimientos sociales, así como en el de la instalación, en Bolivia y Ecuador, de gobiernos que lo reivindican -en un contexto de interculturalidad- como un horizonte civilizatorio

\footnotetext{
${ }^{5}$ Corresponde señalar que si bien la perspectiva de la green economy se funda sobre los postulados de la "economía ortodoxa" también existen, al interior de la disciplina económica, otros planteos acerca de los problemas ecológicos que, superando el reduccionismo de aquella, procuran abordarlos desde un punto de vista holístico. Estos "otros" enfoques "heterodoxos" ponen en discusión toda una serie de presupuestos del mainstream que resultan problemáticos a la hora de abordar los problemas ambientales, tales como la viabilidad del crecimiento económico continuo. Asimismo, apuntan a renovar el arsenal metodológico de la economía orientándolo hacia la interdisciplinariedad, la asunción de la inconmensurabilidad del mundo, el pluralismo valorativo, el rechazo del formalismo matemático, etc. Véase entre otras contribuciones Spash (2009).

${ }^{6}$ Los debates en torno al "vivir bien/buen vivir" han suscitado, en los últimos años, una extensa bibliografía. Como por razones de espacio no podemos citar todos los trabajos publicados hasta la fecha, sólo nos referiremos a aquellos en los que se expresan puntos de vista con los que este artículo discute o bien a aquellas contribuciones que articulan ideas que refuerzan, complementan o desarrollan las que se sostienen aquí.

7 Para una genealogía del buen vivir, remitimos a Aguilar et al. (2015).

${ }^{8}$ Si bien los debates a los que hacemos alusión en este trabajo suelen connotarse a través del uso de diferentes términos y giros -algunos de los cuales proceden de las lenguas de los pueblos indígenas andinos- aquí utilizaremos, de manera uniforme, la expresión "buen vivir" para referirnos a ellos.
} 
y cultural "alternativo" al concepto de desarrollo. Así, el "buen vivir" articula -según una lógica de complementariedad- dimensiones sociales, culturales, políticas, económicas, espirituales, afectivas y ecológicas y aparece como una plataforma para la construcción de nuevas relaciones entre el "capital", la "política", la "sociedad" y la "naturaleza". Sumak kawsay o vida plena, nueva forma de convivencia, "armonía con la naturaleza", etc., son algunos de los ejes que vertebran esas propuestas, las cuales se han visto plasmadas tanto en los sucesivos "planes nacionales" formulados por los gobiernos de Ecuador y Bolivia a partir del año 2007, como en las Constituciones sancionadas en los años $2008^{9}$ y $2009,{ }^{10}$ respectivamente.

Es en el marco de esos debates y reformas en los que emerge un acercamiento alternativo a la cuestión ecológica, ${ }^{11}$ que involucra una serie de desplazamientos respecto del modelo del "desarrollo sustentable" (en sus múltiples formulaciones), y cuyas aristas principales discutimos en este trabajo. ${ }^{12}$

Las contribuciones que, hasta el momento, se han ocupado de la dimensión ecológica del "buen vivir" se han concentrado en resaltar dos de sus rasgos más destacados: la introducción de una posición ética biocéntrica-ecocéntrica y el reconocimiento de derechos a la naturaleza (Gudynas, 2010; Gudynas y Acosta, 2011). Asimismo, se ha tendido a inscribir esa clase de protección en el paradigma de la sustentabilidad, clasificándosela como un modelo de "sustentabilidad super-fuerte" (Gudynas, 2011: 90). Al mismo tiempo, esos análisis se dedicaron a observar críticamente las contradicciones suscitadas tanto en Bolivia como Ecuador, en donde, si por un lado se consagran los derechos de la naturaleza y se promueve su desmercantilización, por el otro se mantienen e intensifican las dinámicas extractivistas que ponen en riesgo el ambiente. Las lecturas generadas en torno a estos conflictos parecen converger en interpretarlos en términos de la distancia existente, por una parte, entre las denuncias realizadas ante las "tribunas internacionales" y la "cotidianeidad nacional" (Gudynas, 2010) y, por otra parte, entre las "constituciones" y los "modelos macroeconómicos" (Viola, 2014).

\footnotetext{
${ }^{9}$ En adelante $\mathrm{CE}$.

${ }^{10}$ En adelante $\mathrm{CB}$.

${ }^{11}$ Como señala Belotti (2014: 43), el concepto de sumak kawsay está irrigado por la disputa ecológica y es a partir de ésta que derivan en cascada las otras dimensiones del significado.

${ }_{12}$ Si bien el aporte que pretendemos realizar en este trabajo consiste en caracterizar los modos en que se aborda la cuestión ecológica en las regulaciones jurídicas y en los planes nacionales que, en Bolivia y en Ecuador, han integrado el punto de vista del "vivir bien/buen vivir", haremos referencia, a lo largo de los diferentes apartados, a algunas de las iniciativas concretas que los gobiernos de Evo Morales y de Rafael Correa han llevado adelante en esa dirección, tanto en el plano nacional como internacional.
} 
Sin desconocer la existencia de las innovaciones éticas y jurídicas marcadas por los autores, la vocación "articuladora" implícita en los planteamientos ambientales realizados en términos de "buen vivir", nila existencia de tensiones, pensamos que esas contribuciones tienden, no obstante, a oscurecer el carácter "multidimensional" y "multiescalar" ${ }^{13}$ que asume ese acercamiento y, en consecuencia, a subestimar tanto los desplazamientos que el mismo introduce respecto del paradigma del "desarrollo sustentable”, como algunos de los desafíos que presenta. Como demostraremos en este artículo, ese acercamiento se expresa en: la convivencia, en el interior del derecho y de los planes nacionales de dichos países, de múltiples significaciones acerca de la naturaleza; la afirmación de la "interdependencia" y la "complementariedad" entre los derechos de la naturaleza y los demás derechos individuales y colectivos reconocidos en esos Estados y la imbricación, en el interior del enfoque del "buen vivir", de significaciones con fuerte "arraigo local" y otras que, por su vocación universal, permiten su proyección "internacional".

Es a partir del reconocimiento de los múltiples puntos de vista y escalas con las que se aborda la cuestión ecológica como, según defendemos en este trabajo, esos planteamientos, en lugar de leerse como reformulaciones "fortificadas" de la idea de "sustentabilidad", pueden emplazarse en un horizonte de inteligibilidad y de acción "otro" respecto del modelo del "desarrollo sustentable". Asimismo, a partir de la asunción de esos rasgos se comprenden mejor las tensiones antes señaladas.

\section{Convivencia de concepciones diversas sobre la naturaleza}

$\mathrm{Al}$ colocar el foco de atención sobre los instrumentos jurídicos e institucionales dispuestos tanto en el interior de las constituciones, como de regulaciones posteriores que abordan la cuestión en términos de "buen vivir", existe cierto acuerdo en considerar que revitalizan la discusión sobre el modo de regular el vínculo naturaleza-sociedad. El punto de partida insoslayable es que tanto la CE (2008) como la Ley n. ${ }^{\circ} 71$ sobre Derechos de la Madre Tierra de $2010^{14}$ y la Ley Marco n. ${ }^{\circ} 300$ sobre Madre Tierra y Desarrollo Integral para Vivir Bien de Bolivia de 2012, ${ }^{15}$ reconocen como sujeto de derecho a la naturaleza, a la Pachamama, a la Madre Tierra.

\footnotetext{
${ }_{13}$ En un artículo en el que compara las propuestas europeas relativas al "decrecimiento" con los planteos del "buen vivir" Unceta (2012: 95) propone interpretar estos últimos a partir de una lógica "multidimensional y multiescalar", sin embargo se trata de una sugerencia que no se explora en el trabajo.

${ }^{14}$ En adelante LDMT.

15 En adelante LMMT.
} 
Este enfoque, centrado en la idea de "buen vivir", implica una ruptura con relación al antropocentrismo que subyace a la idea del "derecho a un ambiente sano" que, si bien se articula con las perspectivas éticas biocéntricas y ecocéntricas que se desarrollaron a lo largo del siglo Xx en diferentes espacios (Esquivel, 2006), trasciende algunas de las discusiones que se dan en esos campos de pensamiento. Al enfocarse en la solidaridad de "lo vivo" y de los elementos que viabilizan la vida, es posible pensar en cierta "igualdad de armas" entre lo humano y lo no humano (Hermitte, 2011) y se re-significan, también, los debates propios del campo de la ética animal en torno a la posibilidad de que todos o algunos animales sean considerados como titulares de derechos. ${ }^{16}$

Así, tanto el artículo 71 de la CE como el artículo 3 de la LDMT encierran concepciones acerca de la naturaleza que involucran la asunción de su valor intrínseco. ${ }^{17} \mathrm{La}$ naturaleza o Pachamama, donde se "reproduce y realiza la vida", tiene derecho a que se respete integralmente su existencia y el mantenimiento y regeneración de sus ciclos vitales, estructura, funciones y procesos evolutivos (CE, art. 71). Asimismo se prescribe que en caso de ser afectada, la naturaleza tiene derecho a la "restauración", la cual es independiente de la obligación que recae sobre el Estado o sobre los particulares, según los casos, de indemnizar a los colectivos e individuos dependientes de los sistemas naturales afectados (CE, art. 72). En relación con lo dispuesto en esta última norma, en el año 2008 se creó, en el ámbito del Ministerio del Ambiente del Ecuador, el "Programa de Reparación Ambiental y Social”, como modo de gestionar los pasivos ambientales derivados de la extracción hidrocarburífera y minera en diversas regiones del país. ${ }^{18} \mathrm{~A}$ través de diferentes cursos de acción, que comprenden aristas sociales y ambientales, se procura contribuir, desde el Estado, a la restitución de los derechos de la naturaleza, de las personas y de las comunidades que viven en las zonas afectadas.

Por su parte, aceptando la recepción de la cosmovisión de los pueblos indígenas, la Madre Tierra se consideraba, en el Estado Plurinacional de Bolivia, sagrada (LDMT, art. 3). En esa dirección, en las leyes procedentes de dicho país se sostiene que ni los componentes ni las funciones y procesos

\footnotetext{
${ }^{16}$ Sobre las principales alineaciones en el interior de este campo disciplinar y sobre la identificación de las propuestas que propugnan o no por una construcción en términos de derechos véase: Afeissa y Jeangène Vilmer (2010).

${ }^{17}$ No puede dejar de observarse, sin embargo, que, como destacan Kareiva y Marvier (2012), entre otros autores, el reconocimiento de que la naturaleza tiene valores extrínsecos no deja de ser una expresión de valores humanos.

${ }^{18}$ En la actualidad se están llevando a cabo planes de reparación integral en las cuencas de los ríos Puyango, Pacayacu y Tenguel.
} 
naturales de la Madre Tierra pueden ser considerados como mercancías, formulación que involucra un punto de ruptura con las propuestas inspiradas en la "economía verde".

Aun cuando existen antecedentes en los que se observa un énfasis en la protección de la naturaleza por su valor intrínseco, ${ }^{19}$ en los sistemas regulatorios a los que nos estamos refiriendo por primera vez se la designa como "sujeto de derecho". Ello trae aparejado una enumeración de los derechos que concede a la naturaleza, como sucede en la legislación boliviana (LDMT, art. 7); de una serie de principios como la "vida en armonía con la naturaleza”, la "no mercantilización", la "interculturalidad” y el "diálogo entre saberes" (LDMT y LMMT); así como un conjunto de "innovaciones institucionales".

En esa dirección, implementando lo dispuesto en el art. 53 de la LMMT a través del Decreto Supremo n. ${ }^{\circ} 1696$ del año 2013, se constituyó, en Bolivia, la Autoridad Plurinacional de la Madre Tierra, una entidad autárquica que funciona bajo la égida del Ministerio de Ambiente y Agua de dicho Estado y es responsable de la formulación de políticas, de la gestión técnica y la ejecución de estrategias relacionadas con los procesos y dinámicas del cambio climático. Su objetivo principal es contribuir a reducir las causas y los efectos de dicho fenómeno, a través de la implementación de plantes que garanticen la capacidad de regeneración de los componentes y sistemas de la vida.

Asimismo, la normativa boliviana de 2012 puso de relieve uno de los grandes temas sobre los que cabe reflexionar en el momento de pensar en el reconocimiento como sujeto de derecho de la Madre Tierra: el problema de su representación y cómo hacer justiciables estos derechos. El título IV de la LMMT se refiere al problema de la legitimación activa para la protección de los derechos reconocidos a la naturaleza y con ese fin enumera tanto a las autoridades públicas, el Ministerio Público, el Tribunal Agroambiental, las personas individuales o colectivas directamente afectadas, como a la Defensoría de la Madre Tierra.

Ciertamente, admitir el valor intrínseco de la naturaleza constituye una forma de resistencia frente a la orientación economicista de la economía verde. Pero la ruptura que el buen vivir representa, de cara al paradigma de la sustentabilidad, estriba, desde nuestro punto de vista, en el reconocimiento simultáneo de "modos plurales" de concebir y valorar la naturaleza

${ }_{19}$ Verbigracia, la Carta Mundial de la Naturaleza aprobada por la Asamblea General de la ONU en 1982 afirma que la especie humana es parte de la naturaleza, que todos los seres vivos poseen un valor intrínseco y merecen respeto. 
y "diversas formas de relacionamiento" con ella. Mientras el enfoque del desarrollo sustentable adopta un punto de vista unidimensional, que encastra desde la mentalidad occidental, el cálculo económico y la racionalidad científica, en Bolivia y Ecuador se organizan regímenes de protección que procuran articular, bajo el horizonte del buen vivir, la valoración intrínseca de la naturaleza, junto a otra serie de significaciones ético-culturales, económicas y políticas. ${ }^{20}$

Así, mientras que, según la CE, la naturaleza es sujeto de derechos, en el Plan Nacional para el Buen Vivir de Ecuador (2009: 100) $)^{21}$ se establece que la naturaleza posee un valor "directo" e "indirecto", siendo fuente de ciencia, tecnología, actividad económica, garantía para la soberanía y seguridad alimentaria y, en el caso de los bosques "fuente de oxígeno con una potencial importancia en el mercado de bonos de carbono a nivel mundial". En el caso de Bolivia, esa concepción multidimensional se expresa, entre otras formulaciones, en el tratamiento que el Plan Nacional de Desarrollo $(2007: 116)^{22}$ le otorga al agua, al considerarla como un "derecho humano, de los demás seres vivos y de la naturaleza”, un "recurso finito” y, en el "contexto cultural $[\ldots]$ como un ser vivo, parte de la naturaleza y proveedor de vida, expresión de flexibilidad, reciprocidad y base del ser humano y su vida [...] un factor de relacionamiento, armonía e integración".

Este esfuerzo por vincular modos plurales de pensar, conocer y relacionarse con la naturaleza, que caracteriza el acercamiento del "buen vivir", no ha pasado totalmente inadvertido ${ }^{23}$ en la literatura. Sin embargo, al enfatizarse la novedad representada por la introducción de la antes

\footnotetext{
${ }^{20}$ Pensamos que los planteos relativos al buen vivir, en la medida en que apuntan a armonizar la vida humana con la protección de la naturaleza, pueden ser puestos en diálogo con la perspectiva de la "ciencia conservacionista" que sustenta Kareiva y Marvier (2012). Estes autores advierten acerca de la imposibilidad de separar la dinámica ecológica de la dinámica humana, enfatizando el hecho de que el destino de la naturaleza y de la humanidad están estrechamente ligados. Así, por ejemplo, tanto la salud como el bienestar humano dependen de la calidad del aire y del agua. A partir de esa clase de razonamientos Kareiva llega a la conclusión de que la conservación no sólo es atendible por el valor intrínseco de la naturaleza sino también por un conjunto de razones prácticas vinculadas con todo aquello que los ecosistemas sanos proveen a la humanidad. Como veremos a lo largo de este apartado, también en el enfoque del buen vivir la protección de la naturaleza encuentra fundamento en un conjunto de razones económicas, geopolíticas y culturales y, nunca, en forma exclusiva, en su valoración intrínseca.

${ }^{21}$ En adelante PNBV 2009.

22 En adelante PNDB 2007

${ }^{23}$ Así Gudynas (2011) alude al buen vivir como una "concepción multidimensional" y afirma que las diferentes significaciones que asume la naturaleza resultan condensadas en el concepto de "patrimonio natural". Sin embargo, no se ocupa de señalar cuáles son esas diferentes dimensiones y, en todo caso, piensa las relaciones entre ellas desde la lógica del "balance" que caracteriza al modelo del desarrollo sustentable, articulando las propuestas del buen vivir como un ejemplo de caso de "sustentabilidad super fuerte".
} 
referida "valoración intrínseca" de la naturaleza, la torsión que dicho enfoque involucra respecto a la "vía única" del desarrollo sustentable ${ }^{24}$ tiende a quedar opacada.

Junto a esa innovación, la naturaleza participa, tanto en los textos constitucionales como en los planes de gobierno de Bolivia y Ecuador, de las apuestas y desafíos económico-sociales, ético-culturales y políticos que comprende, según una perspectiva de "totalidad", el proyecto del buen vivir.

De esta manera, por una parte se resaltan los valores intrínsecos de la naturaleza, que es pensada como la "Pachamama [...] el espacio donde se realiza la vida" (CE, art. 71) y, desde Bolivia, como "la comunidad indivisible de todos los sistemas de la vida y los seres vivos interrelacionados, interdependientes y complementarios, que comparten un destino común" (LMMT, art. 5 inc. 1). A esta aproximación desmarcada del antropocentrismo, con una fuerte impronta comunitaria y trazos vitalistas, se sobrepone, tanto en el caso de la CE como en las leyes sancionadas en Bolivia, el lenguaje moderno y liberal de los "derechos". Se reconoce a la naturaleza como "sujeto colectivo de derecho" (LMMT, art. 5), a la cual se asocia todo un repertorio de derechos; lo que nos induce a pensar en un uso "táctico", con una orientación emancipadora, de ese vocabulario. Pero, por otro lado, la naturaleza también es "objetivada": se la articula en términos de unos "recursos naturales", cognoscibles y susceptibles de apropiación, control, explotación; y cuya significación es tanto económica como política. Sin romper totalmente, con la matriz discursiva de la ortodoxia económica, la biodiversidad se concibe, aun en el interior de los planes nacionales fundados en la idea de buen vivir, como una "ventaja comparativa" (PDNB, 2007: 112 y Plan Nacional de Buen Vivir de Ecuador, 2013: 22)25 con la que contarían los países andinos en el mercado internacional.

Sin embargo, el aprovechamiento -o, más específicamente, la "industrialización”, como se impone en la CB- del patrimonio natural bajo un modelo "ecoeficiente" (PNBV, 2013: 222), que no desdeña la utilización de "incentivos económicos" para fomentar las "mejores prácticas" ambientales, se presenta, al mismo tiempo, como una vía para generar las rentas necesarias para la "transición" hacia otro modo de acumulación, hacia el "biosocialismo" (Le Quang y Vercoutére, 2013).

Así, cuando la aproximación se realiza desde el punto de vista de las políticas de gobierno que persiguen "otro desarrollo", proliferan en los planes

${ }^{24}$ Respecto a la auto-presentación de dicho modelo como una "única respuesta" frente a los problemas ambientales remitimos a Aguilar et al. (2015) y Haidar y Berros (2015).

${ }^{25}$ En adelante, PNBV 2013. 
nacionales de Bolivia y Ecuador las huellas de la racionalidad económica y las referencias a la naturaleza tienden a inscribirse en el paradigma de la "sustentabilidad", según el cual el crecimiento económico debe amoldarse a unos "límites naturales"; expresados, en algunos casos, en términos de la "capacidad de carga de los ecosistemas". En esos cánones, el desarrollo encuentra una limitación que no es política sino pretendidamente "natural" en la capacidad de regeneración de los ecosistemas (se trata, en realidad de un conjunto de definiciones científicas); se apela a la lógica del "balance" / "equilibrio" entre la necesidad de conservación de la naturaleza y las necesidades económicas del desarrollo nacional (PNDB, 2007: 116); el vínculo con la naturaleza tiende a representarse en términos de "gestión" mediada por la utilización de "tecnologías" ambientalmente amigables y, en fin, la etiqueta de "lo sustentable" se sobreimprime a las prácticas productivas y de consumo.

Así, la presencia, en las formulaciones procedentes de los textos jurídicos y de los planes nacionales, de rastros que indican el funcionamiento -en el interior mismo de las propuestas del "buen vivir"- de lenguajes tecnocráticos, semánticas neoliberales y concepciones productivistas, habla de la perdurabilidad y de la enorme capacidad de infiltración de esas matrices (cuyo desmontaje parece resistir a reformas constitucionales y ejercicios de planificación), que "hibridan" las propuestas del buen vivir. Es así que, por ejemplo, se procura garantizar el aprovechamiento "sustentable" de la tierra, mediante estrategias dirigidas desde sectores públicos, privados o comunitarios y se impone como deber al Estado que, en esos usos, se incorporen criterios, no sólo productivos, sino "sociales, ecológicos y espirituales" (LMMT, art.16 inc. 3) o se establece que el Estado promoverá formas de producción que aseguren el buen vivir de la población y "desincentivará" aquellas que atenten contra los derechos de la naturaleza (CE, art. 319).

Reconocer esos rastros -activos al interior del pensamiento y la imaginación política y jurídica- permite poner en funcionamiento, en este sentido, un conjunto de operaciones de pensamiento. Así, cabe entenderlos como pervivencias de formaciones discursivas que se mantienen en el tiempo, imponiéndose aún en el interior de proyectos que se proponen superarlas y que presionando sobre ellos, los traicionan. Pero, también, es posible concebirlos en términos de la apropiación táctica de significantes y prácticas (así, por ejemplo la "eficiencia", la "sustentabilidad", las "tecnologías verdes") que revisten un valor simbólico y/o poseen un conjunto de utilidades que pueden ser encauzadas y re-significadas en el interior de procesos que se auto-presentan como "de transición" y que, junto con (de manera simultánea a) la transformación del vínculo "naturaleza-sociedad", confrontan otras encrucijadas, otras urgencias. 
Es que, al igual que el problema de la explotación de la naturaleza, también la "cuestión racial" y la "cuestión social" están inscritas en las memorias de las luchas de los pueblos de Bolivia y Ecuador y en los proyectos, los debates, etc. relativos al buen vivir. Así, si bien en ambos países tanto los planes nacionales como los procesos de reforma constitucional (mutuamente imbricados) conforman apuestas para colonizar el futuro imaginando alternativas en relación al presente, partieron de la producción de diagnósticos críticos en los que se recuperan las historias de luchas de ambas sociedades y, en lo que a la cuestión ecológica corresponde, afirman la responsabilidad de los países ricos del Norte en la destrucción del planeta.

Así, el modelo del desarrollo sustentable encierra una impronta universalista que oblitera las múltiples relaciones de dominación que atraviesan la cuestión ecológica y subordina el combate contra la pobreza al "crecimiento" conseguido mediante el libre juego de las dinámicas del mercado. Las propuestas que analizamos se alojan, en cambio, en la historia de las luchas contra diversas formas de opresión, articulando, como objetivos "simultáneos", tanto la desmercantilización de la naturaleza como el desmontaje de los dispositivos coloniales y la satisfacción de las necesidades sociales básicas de sus poblaciones, en el marco de proyectos que si bien apuntan a la generación de riqueza (a través del aprovechamiento de los recursos naturales), tienden a ser encauzados por los Estados hacia objetivos de "redistribución" (de bienes y de poderes de decisión) en beneficio de los sectores sociales tradicionalmente excluidos y hacia la construcción del buen vivir como nuevo horizonte civilizatorio y cultural. Es que, como se señala en el PNBV (2009: 19) en sociedades marcadas por la desigualdad no es posible pensar la interculturalidad sin tomar, al mismo tiempo, en cuenta los procesos de dominación. Desde este punto de vista se comprende, también, que la LMMT (art. 45 inc. 2) establezca que las políticas orientadas al vivir bien, incluidas las ambientales, deban ser desarrolladas considerando los procesos de "descolonización" y "despatriarcalización".

Es así como la mirada respecto de la naturaleza asume, también, una significación geopolítica, inscribiéndose en un proyecto que tiende a superar las asimetrías Norte-Sur, mediante, entre otras estrategias, la reafirmación de la soberanía permanente de los Estados sobre los recursos naturales no renovables y la defensa de los recursos naturales en el plano internacional, como pilar de la soberanía. Contemplada desde una perspectiva geopolítica, la naturaleza tiene tanto un valor material como simbólico, tal como se expresa en Ecuador al recuperar la voz de Frantz Fanon (1963): "La Tierra no solo nos dará pan, ante todo la Tierra nos dará dignidad" (PNBV, 2013: 19). 
Por otra parte, desde un punto de vista ético y cultural, la protección de la biodiversidad se vincula, en un contexto de la globalización, con el proyecto de asegurar la pervivencia de otro modo, no occidental, de relacionamiento con la naturaleza, en el marco de una "decisión cultural" como la planteada en el Preámbulo de la CB, de asumir la "pluralidad vigente de todas las cosas".

Desde esa perspectiva, la naturaleza funciona como símbolo del simple "estar": es la tierra para las comunidades rurales, el bosque para las nómades, el barrio y la ciudad para las urbanas (PNDB, 2007: 11). Se busca preservar la naturaleza por su valor intrínseco, pero al mismo tiempo, por la relación "intrínseca" que tienen ciertas comunidades con la naturaleza que genera "visiones de mundo", "interpretaciones del trabajo", "identidades sobre el tiempo y sus mitos", "construcción de la territorialidad y del poder" (ibidem). Así, en el Plan Nacional de Desarrollo de Ecuador (2007: 223$)^{26}$ se enfatiza la existencia de "profundas relaciones entre el patrimonio cultural y natural" y en Bolivia la protección de esas formas de relación con la naturaleza tiene que ver en Bolivia (ibidem) con "preservar y rescatar unas memorias $[\ldots]$ fortalecer otros modos de vida y valores como la complementariedad, la solidaridad, etc.".

Ese modo holístico, mítico, de vincularse con la naturaleza, si bien emergente en las culturas indígenas, persiste bajo diferentes configuraciones en la actualidad; sin circunscribirse, tampoco, a esas culturas. No se apunta a asegurar la conservación de unas prácticas tradicionales a la manera de reliquias: por el contrario, el significante "tradición" no está connotado en los planes nacionales de Bolivia y Ecuador de forma unívoca, ni se asocia a determinados ámbitos de prácticas sino que circula. Si por un lado califica, de manera negativa, las concepciones neoliberales de desarrollo, por otro lado, "pluralizado" (así "tradiciones") se utiliza como sinónimo de "modos de vida".

La aproximación holística a la naturaleza contradice la concepción moderna y capitalista que funda la relación sobre la "separación" naturaleza/ sociedad y la "objetivación" de ambas, e involucra un desplazamiento desde una visión de mundo basada en el "ser" a otra fundada en el "estar siendo" (Kusch, 2012 [1976]). En sí misma, y por la aportación -a la vez crítica y constructiva- que el tipo de experiencia existencial que ella supone puede hacer a Occidente, esa relación mítica con la naturaleza se considera valiosa, en el horizonte de plurinacionalidad e interculturalidad en construcción que caracteriza a Bolivia y Ecuador. Sin embargo, la decisión de proteger ese otro

${ }^{26}$ En adelante PNDE 2007. 
modo de relacionarse con la naturaleza, no deja de estar tamizado, en cierto sentido, por el espíritu del comercio internacional. Así, la interculturalidad se lee, en el caso del PNDE 2007 como una "potencialidad" y una "oportunidad" de un país periférico en el mundo globalizado, mientras que en el PNDB (2007: 11) se afirma que "los sólidos vínculos de las culturas bolivianas con la naturaleza son un patrimonio de todos y constituye una enorme ventaja comparativa en relación a un modelo de desarrollo capitalista".

Al reconocerse esos "vínculos" -cuya solidez se basa en su carácter mítico- la racionalidad científica moderna pierde su "monopolio" como ámbito exclusivo de producción de verdad y se legitiman los saberes ancestrales que son patrimonio de los pueblos indígenas; proponiéndose estrategias fundadas en el "diálogo de saberes". Hay un desplazamiento desde la dicotomía hacia la diversidad, lo cual genera el interrogante relativo a las formas en que se imaginan las "relaciones" entre esos modos plurales de concebir la naturaleza. Como veremos en el apartado siguiente, la circunstancia de que en Ecuador y Bolivia los derechos de la naturaleza convivan con otros derechos tanto de los "individuos" como de las "comunidades" y los "pueblos", hace particularmente relevante dicha pregunta.

\section{Los desafíos de la complementariedad entre derechos}

Uno de los desafíos que plantea el enfoque ecológico del buen vivir es que los derechos de la naturaleza coexisten, en el interior del ordenamiento jurídico, con otros repertorios de derechos "individuales" y "colectivos". ${ }^{27}$ Esta situación se presenta, en el caso del Ecuador, en el interior del propio texto constitucional, mientras que en Bolivia una convivencia semejante se da en el texto de la LMMT. La circunstancia de que, junto a los derechos de la naturaleza (que, es preciso recordar, en Bolivia están desprovistos de jerarquía constitucional), ${ }^{28}$ se reconozca a las personas, las comunidades, pueblos y nacionalidades el derecho a beneficiarse del ambiente y de las riquezas naturales que les permitan el buen vivir (CE, art. 74) o, como en Bolivia, se imponga al Estado el deber de industrializar los recursos naturales, puede suscitar toda una serie de conflictos.

Esas tensiones -entre otras posibles- se recortan en el marco de las políticas económicas neo-extractivistas desarrolladas como vías de "transición"

\footnotetext{
${ }^{27}$ El universo de "sujetos de derechos" que reconoce la CE incluye: a las personas, las comunidades, los pueblos, las nacionalidades, los colectivos y la naturaleza (art. 10).

${ }^{28}$ Si bien los "derechos de la naturaleza" fueron reconocidos en ese país en dos leyes, la circunstancia de que el principio del "vivir bien" tenga estatuto constitucional habilita la posibilidad de interpretaciones jurisprudenciales que entiendan que los derechos de la naturaleza están asociados, como normas fundamentales con rango constitucional, a ese principio.
} 
hacia el buen vivir, según la idea de que la utilización intensiva de los recursos naturales permitiría resolver las situaciones de desigualdad, consideradas prioritarias. Así, mientras la LMMT (art. 4 inc. 11) establece que, en el contexto de las estrategias de desarrollo, el Estado otorga prioridad a las personas de menores ingresos económicos y con mayores problemas en la satisfacción de sus necesidades materiales, sociales y espirituales y goce pleno de sus derechos fundamentales, en el PNBV (2013: 24) se señala que "el primer paso es resolver el acceso a bienes, oportunidades y condiciones que le garanticen -al individuo, a la colectividad y a las generaciones futuras- una vida digna [si bien se aclara] sin perjudicar a la naturaleza". ${ }^{29}$

Encrucijadas de este estilo entre la "protección de la naturaleza", el "desarrollo" y la "lucha contra la pobreza", atraviesan, desde su propia emergencia, la cuestión ecológica. Frente a ellas, el modelo del desarrollo sostenible, se ha inclinado -en el registro de la política internacional y no del derecho- hacia una estrategia de "conciliación". Siguiendo este espíritu, en los diferentes países que, a lo largo de las últimas décadas, incorporaron derechos ambientales a sus textos constitucionales, la lógica del "balance" se ha expresado, en las decisiones judiciales que deben resolver los conflictos entre derechos, en la utilización de estrategias de "ponderación" entre principios. Así, aun cuando en un plano "abstracto" todos los derechos fundamentales tienen el mismo rango, en los casos concretos en los que se presentan "colisiones" se admite que los derechos tienen distinto "peso" y que uno -el que tiene más "peso"- debe "ceder" ante el otro (Alexy, 1997: 89).

Fundamentado en una teoría liberal de los derechos, y pensado para resolver conflictos entre derechos de los "individuos", el procedimiento de la "ponderación" no parece compadecerse, sin embargo, con la filosofía social que subyace al buen vivir. Así, ni la lógica binaria que establece que un derecho debe "ceder" frente a otro, ni el tipo de razonamiento cuantitativo (un derecho "pesa" más que otro) que están implícitos en esa clase de estrategia se corresponde con el conjunto de valores sociales en torno a los cuales se edifica el buen vivir. Por el contrario, la solidaridad, complementación, co-responsabilidad, subyacen a la demarcación de algunos criterios para la resolución de conflictos en los que aparece como medular la no afectación de los diferentes sistemas de vida (LDMT, art. 6).

\footnotetext{
${ }^{29}$ Los problemas que pueden derivarse del aprovechamiento económico de la naturaleza no agotan, no obstante, el caudal de potenciales conflictos que se derivan del reconocimiento simultáneo de diferentes derechos (sociales, económicos, culturales, etc.; individuales "y" colectivos) a una multiplicidad de "sujetos jurídicos".
} 
La afirmación de esos valores significa poner en discusión los del formato liberal en los que, sin embargo, están inscritos los proyectos emancipatorios planteados desde Bolivia y Ecuador (Dávalos, 2013).

De manera aun más precisa, los razonamientos que se enmarcan en el modelo de la "ponderación" no se adecuan a la "regla hermenéutica" de la "interdependencia" y la "complementariedad" que introduce el modelo del buen vivir, para organizar la convivencia -y los posibles conflictos- entre "derechos", "obligaciones" y "deberes". Así, mientras en las Constituciones de ambos Estados se enfatiza la idea de la "interdependencia" entre los derechos, la LMMT la desarrolla a través de formulaciones que establecen que "un derecho no puede materializarse sin los otros o no puede estar sobre los otros, implicando la interdependencia y apoyo mutuo" (art. 4 inc. 1) y que afirman la "interrelación, interdependencia y funcionalidad" de todos los aspectos y procesos sociales, culturales, ecológicos, económicos, productivos, políticos y afectivos (art. 4 inc. 3 ).

Asimismo, imbricados con los procesos constituyentes y las reformas jurídicas impulsadas en ambos países por los gobiernos de Evo Morales y Rafael Correa, los planes nacionales insisten sobre la perspectiva de la "complementariedad", idea que, como destaca Tapia (2011), está fuertemente ligada a la de "redistribución".

Es que, como ha sido destacado en numerosos análisis (Larrea, 2010), el "buen vivir" supone una cosmovisión holista, que establece la complementariedad de todos los seres vivos, formas de conocimiento (así, entre las "ciencias" y los "saberes ancestrales" y "tradicionales"), modos productivos, etc. Si en el ámbito de la "política", ${ }^{30}$ esa idea abre el juego a las "negociaciones" permanentes para "construir nuevas síntesis, lograr una compresión plural de la realidad" como se señalara en el PNDE (2007: 221), traducida al campo jurídico, esa mirada plantea, como desafío, la superación de los razonamientos basados en la "ponderación" entre derechos y la invención de nuevas hermenéuticas, orientadas hacia la realización simultánea y acompasada de los diversos derechos. Este esfuerzo de compatibilización exigirá la revisión y reelaboración de instituciones clásicas del derecho privado, tales como la "propiedad" y el "contrato". Avanzando en esa dirección, la CE (art. 321) establece que el derecho de propiedad, en todas sus formas, deberá cumplir su "función social y ambiental".

${ }^{30}$ Espacio al que, por otra parte, también Gudynas (2011) reenvía los conflictos que pueden plantearse entre las diversas significaciones que asume la naturaleza en el interior del enfoque de la sustentabilidad super-fuerte. 
No puede dejar de destacarse, por otra parte, que con la idea de buen vivir, se enfatiza y se pone en valor una perspectiva de "totalidad" que, ciertamente, es afín al pensamiento mítico característico de la cosmovisión de los pueblos andinos pero que no deja de estar presente, tampoco, en los actuales esfuerzos de "planificación nacional" ensayados desde ambos países, que evocan una racionalidad socialista a inventarse. Así, el "nosotros" es conjugado, en los proyectos que estamos analizando, en varias formas -míticas y discursivas- y lenguas, que incluyen frecuentes referencias a "lo comunitario", pero, asimismo a "lo nacional" y a lo "estatal": el dispositivo de la ciudadanía y del "pacto social" como respuestas a la interrogación por la "totalidad" con la figura de la "comunidad", a través de la cual se expresa la utopía de la des-alienación: "Vivir bien como parte de la comunidad" [...] significa vivir bien contigo y conmigo lo cual es diferente del "vivir bien" occidental que es individual, separado de los demás e inclusive a expensas de los demás y separado de la naturaleza (PNDB, 2007: 10). "Compartimos [...] el 'estar' juntos con todos los seres, que tienen vida y son parte de la nuestra. El mundo de arriba, el mundo de abajo, el mundo de afuera y el mundo del aquí, se conectan y hacen parte de esta totalidad, dentro de una perspectiva espiral del tiempo no lineal" (PNBV, 2009: 18).

En consonancia con la exaltación de la dimensión "común" de la existencia y aun cuando las Constituciones no establecen jerarquías entre derechos, no deja de insinuarse, en el derecho y en los planes nacionales, la decisión de priorizar lo colectivo sobre lo individual. En esta dirección, la propia CE (art. 83 inc. 7) impone como deber y responsabilidad de los ciudadanos "promover el bien común y anteponer el interés general al interés particular, conforme al buen vivir" y ello "da cuenta" según la interpretación ensayada en el PNBV (2013: 32) de la "primacía de lo colectivo sobre lo individual".

\section{La dimensión multiescalar del buen vivir: su proyección hacia el espacio global}

Los planteamientos relativos al buen vivir si bien tienen un fuerte arraigo local, trascienden el plano de las culturas andinas para proyectarse sobre el ámbito global. En este sentido, es posible reconocer en estas propuestas varias escalas de incumbencia, una inscripción de modos de vida enraizados en unas comunidades locales en discursos, instrumentos jurídicos e instituciones que hacen a la construcción de "lo nacional" y, a su vez, cierta proyección de las propuestas nacionales pluriculturales hacia el ámbito internacional.

Por un lado, los instrumentos jurídicos que protegen a la naturaleza como un bien en sí -atribuyéndole la categoría de "sujeto de derecho"y postulan modos de vida en armonía con la naturaleza están consagrados 
en la Constitución de Ecuador y en legislación de Bolivia. Esta inscripción constitucional del buen vivir ha sido leída como una tentativa de traducir un concepto local en términos universales, a través del lenguaje de los derechos, inteligible para la cultura jurídica moderna (Belotti, 2014).

A su vez, los procesos de reformas constitucionales y de producción de leyes están imbricados con los planes "nacionales" de ambos Estados; en una relación dialéctica entre "derecho" y "política", en función de la cual la planificación nacional aparece como medio para la realización y garantía de los derechos.

No puede soslayarse que esos planes y esas constituciones y leyes son el emergente de un conjunto de procesos sociales que involucraron la recuperación, la relectura y la recreación de las historias de esos países y de las memorias plurales de sus pueblos y comunidades. Tampoco que, en continuidad con la larga tradición de lucha anticolonialista de los países del Tercer Mundo, uno de los ejes del modelo alternativo de regulación de la cuestión ecológica esbozado desde Bolivia y Ecuador es la soberanía nacional sobre los recursos naturales.

Pero ese conjunto de inscripciones que dan cuenta de la filiación del enfoque del buen vivir en unos modos de existencia históricos y singulares, en una "cultura (pluri)nacional" no clausuran su significación. Por el contrario, esa perspectiva también tiene una vocación universalista. ${ }^{31}$ En este sentido, las políticas internacionales desplegadas por los gobiernos de Evo Morales y Rafael Correa, tanto en el sistema de Naciones Unidas como en otros foros mundiales, han tendido a impulsar la traducción del lenguaje, la ética y los instrumentos jurídicos que establecen, en sus países, un modo de protección de la naturaleza alternativo a la perspectiva de la economía verde, en los documentos que organizan el régimen de gobernanza global del ambiente. Y, al mismo tiempo, han buscado responsabilizar moral y jurídicamente, así como implicar en términos políticos y económicos a los Estados del Norte, en la protección de la naturaleza.

En este sentido, entre los cursos de acción con trascendencia internacional, no puede dejar de señalarse la iniciativa Yasuní-ITT. ${ }^{32}$ Propuesto por el gobierno de Correa en junio de 2007 y presentada en septiembre

\footnotetext{
${ }^{31}$ Cf. con Viola (2014: 68) quien sostiene que el valor potencial del sumak kawsay reside solamente en su carácter "local" en su arraigo en un contexto socio-cultural concreto.

32 Yasuní es un Parque Nacional que se encuentra al límite de la Alta Amazonía y de la Cordillera de los Andes. La UNESCO lo declaró en 1989 reserva mundial de la biosfera en virtud de la excepcional biodiversidad que alberga. Las siglas "ITT" hacen a alusión a los yacimientos petrolíferos Ishpingo, Tambococha y Tiputini que se encuentran en dicho Parque. Para un desarrollo in extenso del tema remitimos a Le Quang, 2013.
} 
de ese mismo año en la Asamblea de la ONU, dicho proyecto consistía en dejar bajo tierra lo equivalente al 20\% de las reservas de petróleo del Ecuador, siempre que los países históricamente responsables de los cambios climáticos accedieran a aportar una contribución financiera equivalente a la mitad de las rentas que el Estado hubiera obtenido en el caso de llevar adelante la explotación. ${ }^{33}$ Con ello se buscaba no sólo evitar la emisión de gases contaminantes a la atmósfera, sino conservar uno de los ecosistemas más biodiversos del planeta y respetar los derechos de los pueblos indígenas que viven en el Parque Yasuní.

Ese aporte financiero solicitado por el Ecuador a los países desarrollados -los cuales pasarían a constituir un fondo fiduciario administrado por el PNUD-, se relacionaba, de manera directa, con las políticas internacionales orientadas a la realización de la justicia ecológica, que tanto dicho país, como Bolivia, llevan adelante en distintos foros. Dicha iniciativa, que se convirtió en emblema de un planteamiento alternativo respecto de la cuestión ecológica, fue finalmente dada de baja en el año 2013 frente a la falta de integración de los fondos solicitados.

Así, desde hace algunos años estas perspectivas circulan en un espacio que trasciende los límites locales y nacionales, en un movimiento que tiende a subvertir las relaciones de dependencia Norte-Sur, a las que no han sido ajenos los modos de regular el problema ambiental (Haidar y Berros, 2015). Una de las primeras huellas que el enfoque del buen vivir ha dejado en el ámbito internacional es la Resolución n. ${ }^{\circ}$ 63/278 de Naciones Unidas que, en 2009 y a iniciativa de Bolivia, fijó el día 22 de abril como Día Internacional de la Madre Tierra. A ella le han seguido una serie de resoluciones que conciernen a la vida en "armonía con la naturaleza". Subyace a esa formulación una lucha por la construcción del sentido, que oscila entre la perspectiva que, de la mano del vivir bien, reconoce a la Madre Tierra, la Pachamama, como sujeto de derecho y la inscripción de esa idea como un tópico del paradigma del desarrollo sostenible.

Este tipo de tensiones no sólo se verifican al interior de la serie de resoluciones mencionadas. En la reciente discusión sobre la "puesta en práctica" de Rio + 20 en el seno del Programa de Naciones Unidas para el Medio Ambiente, desarrollada en Nairobi en 2013, existió un debate en este sentido, sostenido, también, por Bolivia. De la discusión sobre el rol del Programa de Naciones

\footnotetext{
${ }^{33}$ Las grandes líneas del proyecto Yasuní fueron discutidas por diferentes asociaciones ecologistas, movimientos sociales, organizaciones indígenas antes de que Rafael Correa llegara al poder. Desde esos espacios se planteó la necesidad de decretar una "moratoria" en la expansión de la frontera petrolera de la Amazonía (véase Acosta et al., 2009).
} 
Unidas para poner en práctica la economía verde se llegó a un acuerdo en el que se reconoce que ésta es una de las alternativas posibles y que, además, existen otros enfoques, modelos y herramientas como el vivir bien en armonía con la Madre Tierra: Entonces en el mundo se ha empezado a asumir que ya no existe sólo la vía básicamente eurocéntrica de la economía verde sino también su antítesis que es la del "Vivir Bien en armonía y equilibrio con la Madre Tierra" (Pacheco, 2013).

Esto no hace sino reeditar lo que, desde Bolivia, se viene sosteniendo desde 2010 cuando, frente al fracaso de las negociaciones sobre cambio climático en Copenhague, se propulsó una Declaración sobre Cambio Climático y Derechos de la Madre Tierra aprobada por 35000 personas en Cochabamba que contiene algunos de los elementos cardinales para pensar que la economía verde no contiene la totalidad de las respuestas frente a la crisis ecológica.

Asimismo, con la mirada puesta en la Convención de Rio + 20, la estrategia de juridificación internacional del punto de vista de la Madre Tierra fue introducida en la Resolución n. ${ }^{\circ}$ 66/204 de 2011 donde se recomendaba "Considerar la posibilidad de formular una declaración en que se reconozca el valor intrínseco de la naturaleza y de su capacidad regeneradora en el contexto de la Conferencia de Naciones Unidas sobre Desarrollo Sostenible de junio de 2012". Si bien Rio + 20 no materializó la anhelada declaración, se registran algunas huellas de esas posiciones tanto en los denominados "zero drafts" elaborados por el grupo de países del Tercer Mundo que integran Ecuador y Bolivia, así como en el documento final de dicha Conferencia.

En esas iniciativas internacionales de los países andinos se expresa la dimensión "cosmopolita" que, sin perjuicio del anclaje "nacional" y "local", asume el modo de abordaje de la cuestión ecológica que caracteriza a las propuestas fundadas en la idea de buen vivir. Dicha vocación universalista se corresponde con una concepción de la naturaleza en términos de un "patrimonio común de todos los seres vivos existentes" (Morales, 2012) pero convive, como hemos señalado anteriormente, con otras significaciones. Desde esa perspectiva, los derechos de la naturaleza, el reconocimiento del "valor de uso" de las cosas y de las acciones, así como la preservación de la relación "intrínseca" con la naturaleza propia del modo de vida de los pueblos indígenas (pero que no deja de reaparecer, como trasfondo y residuo mítico marginal en el modo de vida occidental) es el "aporte" que los países del Sur realizan a la "comunidad internacional", con la finalidad de confrontar la crisis civilizatoria y de avanzar hacia la realización de la justicia social y ecológica. 


\section{A modo de conclusión}

En este artículo nos concentramos en analizar las múltiples dimensiones y escalas que reviste el modo en que, desde el punto de vista del buen vivir, se aborda en Ecuador y Bolivia la cuestión ecológica. Hemos sostenido que la convivencia, en estos planteamientos, de significaciones plurales acerca de la naturaleza así como la circunstancia de que dichas propuestas se articulen, a la vez, en varias espacialidades, obedecen a varios factores: la decisión cultural de preservar y proteger un modo de vida no occidental, la heterogeneidad del universo de discursos en los que se alojan dichas propuestas (en el que conviven y no sin dificultad, el liberalismo político, ciertas marcas de la ortodoxia económica y cosmovisiones fundadas en el pensamiento mítico, entre otras matrices), así como a las encrucijadas históricas que atraviesan a esas sociedades y que los gobiernos de Evo Morales y Rafael Correa procuran confrontar.

Afirmamos que en esa pluralización de significados, estrategias y espacios que caracteriza el modo de confrontarse la cuestión ecológica, reside la ruptura que la perspectiva del buen vivir produce respecto del modelo (que se articula como unívoco) del "desarrollo sustentable". Ello impide considerarla (y aun cuando explote una semántica economicista y hable, por momentos, un lenguaje tecnocrático) como una reformulación más del paradigma de la sustentabilidad.

Asimismo, hemos planteado que el reconocimiento de ese carácter "multidimensional" y "multiescalar" coadyuva a plantear adecuadamente las críticas y limitaciones que presentan, en la actualidad, esas propuestas, las cuales no radican en su supuesto "juridicismo" (Viola, 2014) ni se explican en términos de la oposición "retórica internacional/práctica nacional" sino que se vinculan, más bien, con las dificultades que se derivan de la yuxtaposición de distintos "lenguajes" y "formatos" y con la insuficiente elaboración de la que adolece, en el marco de los debates sobre el buen vivir, la cuestión de la "complementariedad".

En esta dirección, el buen vivir se presenta como una "exploración de alternativas" (Gudynas y Acosta, 2011: 78) tanto respecto a la idea de "desarrollo" como también en lo que respecta a las modalidades de relacionamiento entre concepciones distintas acerca de la naturaleza, donde el desafío está planteado, desde nuestro punto de vista, por superar los modelos liberales del "intercambio" y la "ponderación". En ese espacio se inscriben importantes tareas para el pensamiento socio-jurídico, al que le cabe, entre otras, la misión de señalar las vías por medio de las cuales la perspectiva de totalidad que subyace a toda cultura (y, particularmente, a aquellas que defienden la "pluralidad" en un sentido fuerte) se aloja e 
irrumpe en el campo del derecho, presionando hacia la imaginación y la institucionalización de estrategias fundadas en la complementariedad y en el diálogo entre lo radicalmente diferente.

\section{Revisión de Eloísa Álvarez}

\section{Referencias bibliográficas}

Acosta, Alberto (2012), Sumak kawsay. Una oportunidad para imaginar otros mundos. Quito: Abya Yala.

Acosta, Alberto; Gudynas, Eduardo; Martínez, Esperanza; Vogel, Joseph (2009), “Dejar el crudo en tierra o la búsqueda del paraíso perdido. Elementos para una propuesta política y económica para la Iniciativa de no explotación del crudo del ITT", Polis: revista académica de la Universidad Bolivariana, 8(23), 429-452.

Afeissa, Hicham-Stéphane; Jeangène Vilmer, Jean-Baptiste (comps.) (2010), "Étique animale. Introduction”, in Philosophie animale. Différence, responsabilité, communauté. Paris: Librairie Philosophique Vrin, 3-6.

Aguilar, Paula; Fiuza, Pilar; Glozman, Mara; Grondona, Ana; Haidar, Victoria; Pryluka, Pablo (2015), "Hacia una genealogía del buen vivir: contribuciones desde el análisis materialista del discurso", Theomai Estudios Críticos sobre Sociedad y Desarrollo, 32 , en prensa.

Alexy, Robert (1997), Teoría de los derechos fundamentales. Madrid: Centro de Estudios Constitucionales.

Belotti, Francesca (2014), "Entre bien común y buen vivir. Afinidades a distancia", Íconos. Revista de Ciencias Sociales, 48, 41-54.

Courtine, Jean Jacques (1981), "Quelques problèmes théoriques et méthodologiques en analyse du discours, à propos du discours communiste adressé aux chrétiens”, Langages, 15(62), 9-128.

Dávalos, Pablo (2013), "La pluralidad del Estado y las aporías del liberalismo", América Latina en Movimiento online - ALAI-Agencia Latinoamericana de Información, 23 de julio. Consultado el 12.02.2015, en http://www.alainet.org/es/active/65914. Esquivel Frías, Eleonora (2006), Responsabilidad y sostenibilidad ecológica. Tesis Doctoral presentada a la Facultad de Filosofía y Letras de la Universidad Autónoma de Barcelona, Barcelona, España.

Gudynas, Eduardo (2010), "La senda biocéntrica: valores intrínsecos, derechos de la naturaleza y justicia ecológica”, Tabula Rasa, 13, 45-71.

Gudynas, Eduardo (2011), "Desarrollo, derechos de la naturaleza y buen vivir después de Montecristi”, in Gabriela Weber (comp.), Debates sobre cooperación y modelos de desarrollo. Perspectivas desde la sociedad civil en Ecuador. Quito: Centro de Investigaciones Ciudad y Observatorio de la Cooperación al Desarrollo, 83-103. 
Gudynas, Eduardo; Acosta, Alberto (2011), “La renovación de la crítica al desarrollo y el buen vivir como alternativa”, Utopía y Praxis Latinoamericana, 53,71-83.

Haidar, Victoria; Berros, Valeria (2015), "Entre el sumak kawsay y la 'vida en armonía con la naturaleza': disputas en la circulación y traducción de perspectivas respecto de la regulación de la cuestión ecológica en el espacio global", Theomai Estudios Críticos sobre Sociedad y Desarrollo, 32, en prensa.

Hermitte, Marie-Angèle (2011), "La nature, sujet de droit?”, Annales. Histoire Sciences Sociales, 1-2011, 173-212.

Jacobs, Michael (1991), The Green Economy. Environment, Sustainable Development and the Politics of the Future. London: Pluto Press.

Kareiva, Peter; Marvier, Michelle (2012), "What is Conservation Science”, BioScience, 62, 962-969.

Kusch, Rodolfo (2012), Geocultura del bombre americano. Rosario: Fundación Ross [ed. orig.: 1976].

Larrea, Ana María (2010), "La disputa de sentidos por el Buen Vivir como proceso contrahegemónico", in SENPLADES/IAEN/MCP (comps.), Los nuevos retos de América Latina. Socialismo y sumak kawsay. Quito: SENPLADES, 15-28.

Le Quang, Mathieu (2013), Dejar el petróleo bajo tierra. Quito: IAEN.

Le Quang, Mathieu;Vercoutére, Tamia (2013), Ecosocialismo y buen vivir. Diálogo entre dos alternativas al capitalismo. Quito: IAEN.

Milani, Brian (2000), Designing the Green Economy. The Postindustrial Alternative to Corporate Globalization. Boston: Rowman \& Littlefield Publishers.

Morales, Evo (2012), “Discurso de Evo Morales Ayma, Presidente del Estado Plurinacional de Bolivia”, Plenaria de la Conferencia de Naciones Unidas sobre Desarrollo Sostenible Rio + 20, Rio de Janeiro, Brasil, 21 de junio de 2012.

Pacheco, Diego (2013), “Enfoque 'Vivir Bien' es reconocido internacionalmente”, América Latina en Movimiento online - ALAI-Agencia Latinoamericana de Información, 18 de marzo. Consultado el 12.02.2015, en http://www.alainet.org/es/active/62572.

Rajagopal, Balakrishnan (2003), International Law from Below. Cambridge: Cambridge University Press.

Santos, Boaventura de Sousa (2010), Refundación del Estado en América Latina. Perspectivas desde una epistemología del sur. Quito: Abya Yala.

Spash, Clive (2009), "Social Ecological Economics", Socio-economics and the Environment in Discussion. Canberra: CSIRO Working Paper Series.

Tapia Mealla, Luis (2011), "Una geopolítica de la complementariedad”, in Ivonne Farah H.; Luciano Vasapollo (coords.), Vivir bien. ¿Paradigma no capitalista? Bolivia: CIDES-UMSA, 383-400.

Unceta, Koldo (2012), “Crecimiento, decrecimiento y buen vivir”, in Alejandro Guillén García; Mauricio Phélan Casanova (comps.),Construyendo el buen vivir. Ecuador: Universidad de Cuenca, 85-98. 
Viola Recanses, Andreu (2014), “Discursos 'pachamamistas' versus políticas desarrollistas: el debate sobre el sumak kawsay en los Andes”, Ícono, 48, 55-72.

\section{Documentos de gobierno y normas jurídicas citadas}

CB - Constitución del Estado Plurinacional de Bolivia, 2009.

CE - Constitución de Ecuador, 2008.

Declaración Universal Derechos de la Madre Tierra, Cochabamba, 2010.

Documento Final de la Conferencia de Naciones Unidas sobre Desarrollo Sostenible "El futuro que queremos", Rio+20, 2012.

LDMT - Ley de Derecho de la Madre Tierra n. ${ }^{\circ}$ 71, Bolivia, 2010.

LMMT - Ley Marco de la Madre Tierra y Desarrollo Integral para el Vivir Bien n. ${ }^{\circ} 300$, Bolivia, 2012.

Informe "Nuestro Futuro Común”-Reporte Brundtland, 1987.

PNDB - Plan Nacional de Desarrollo: Bolivia digna, soberana, productiva y democrática para Vivir Bien, 2007.

PNDE - Plan Nacional de Desarrollo, Ecuador, 2007-2010.

PNBV - Plan Nacional para el Buen Vivir, Ecuador, 2009-2013.

PNBV - Plan Nacional Buen Vivir, Ecuador, 2013-2017.

Resolución ONU “Armonía con la Naturaleza”, n. ' 66/204, 2011.

Recibido: 30.03.2015

Aceptación comunicada: 19.10.2015

\section{Victoria Haidar}

Consejo Nacional de Investigaciones Científicas y Técnicas, Centro de Investigaciones de la Facultad de Ciencias Jurídicas y Sociales de la Universidad Nacional del Litoral

Cándido Pujato 2751, S3002ADG Santa Fe, Argentina

Contacto: vhaidar@fcjs.unl.edu.ar

\section{María Valeria Berros}

Centro de Investigaciones de la Facultad de Ciencias Jurídicas y Sociales de la Universidad Nacional del Litoral

Cándido Pujato 2751, S3002ADG Santa Fe, Argentina

Contacto: vberros@fcjs.unl.edu.ar 
Towards a Multidimensional and Multi-scale Approach of the Ecological Issue:

The Perspective of Good Living

This article proposes to replenish the multidimensional and multi-scale character of the ecological issue embodied in the "good living" proposals of Ecuador and Bolivia by focusing on three aspects: i) the multiple meanings of nature that coexist in the regulations and national plans of both countries; ii) the statement about the interdependence and complementarity of the rights of nature relative to individual and collective rights, and iii) the circulation of these proposals from Latin America's Andean region in the global arena.

Keywords: Bolivia; ecology; Ecuador; good living; environmental protection.
Vers un abordage multidimensionnel et multi-échelle de la question écologique: La perspective du "bon vivre"

Cet article se propose de restituer le caractère multidimensionnel et multi-échelle assumé par la question écologique à l'intérieur de la proposition du "bon vivre" en Équateur et en Bolivie à partir de trois axes: i) les multiples significations de la nature qui coexistent dans les régulations et dans les plans nationaux des deux pays, ii) l'affirmation de l'interdépendance et de la complémentarité des droits reconnus de la nature par rapport aux droits individuels et collectifs, iii) la circulation de ces propositions depuis la région andine de l'Amérique latine vers le reste du monde. Mots-clés: Bolivie; bon vivre; écologie; Équateur; protection de l'environnement. 